\title{
Lattices and quantales of ideals of semigroups and their preservation under Morita contexts
}

\author{
Valdis Laan, László Márki and Ülo Reimaa
}

\begin{abstract}
We study properties of the lattice of unitary ideals of a semigroup. In particular, we show that it is a quantale. We prove that if two semigroups are connected by an acceptable Morita context then there is an isomorphism between the quantales of unitary ideals of these semigroups. Moreover, factorisable ideals corresponding to each other under this isomorphism are strongly Morita equivalent.
\end{abstract}

Mathematics Subject Classification. 20M30, 20M50.

Keywords. Semigroup, Ideal, Morita context, Quantale.

\section{Introduction}

Just like in rings, Morita contexts have turned out to be very useful for studying Morita equivalence of semigroups as well. Using Morita contexts it is possible to prove that many properties are shared by all semigroups in the same Morita equivalence class (such properties are said to be Morita invariant). In our earlier paper [4] we proved that the ideal lattices of Morita equivalent semigroups with weak local units are isomorphic. However, the question if this holds also for larger classes of semigroups remained open. In the present article we show that the ideal lattices of Morita equivalent firm semigroups need not be isomorphic. On the other hand, it turns out that considering unitary ideals instead of all ideals it is possible to prove the isomorphism of the corresponding lattices (actually even quantales) under much weaker assumptions - putting no

\section{Presented by Tim Stokes.}

The research of V. Laan was supported by the Estonian Research Council grant PUT1519. The research of L. Márki was partially supported by the Hungarian National Research, Development and Innovation Office, NKFIH, grant no. K119934. The research of Ü. Reimaa was supported by the Estonian Research Council grants PRG49, PSG114 and PUT1519. Mutual visits of the authors were made possible by the exchange agreement between the Estonian and the Hungarian Academies of Sciences. 
assumptions on the semigroups and requiring only acceptability for the context that connects the two semigroups.

In this paper, semigroups and ideals are allowed to be empty and $S, T$ will always stand for semigroups.

A right $S$-act $A_{S}$ is said to be unitary (firm) if the mapping $A \otimes S \rightarrow$ $A, a \otimes s \mapsto a s$ is surjective (bijective). Thus $A_{S}$ is unitary if and only if $A S=A$. A biact ${ }_{S} A_{T}$ is called unitary if $S A=A=A T$. A semigroup $S$ is said to be factorisable (firm) if the act $S_{S}$ is unitary (firm).

Definition 1.1. A Morita context is a six-tuple $\left(S, T,{ }_{S} P_{T},{ }_{T} Q Q_{S}, \theta, \phi\right)$, where $S$ and $T$ are semigroups, ${ }_{S} P_{T}$ is an $(S, T)$-biact and ${ }_{T} Q_{S}$ is a $(T, S)$-biact, and

$$
\theta:{ }_{S}\left(P \otimes_{T} Q\right)_{S} \rightarrow{ }_{S} S_{S}, \quad \phi:{ }_{T}\left(Q \otimes_{S} P\right)_{T} \rightarrow{ }_{T} T_{T}
$$

are biact morphisms such that, for every $p, p^{\prime} \in P$ and $q, q^{\prime} \in Q$,

$$
\theta(p \otimes q) p^{\prime}=p \phi\left(q \otimes p^{\prime}\right), \quad q \theta\left(p \otimes q^{\prime}\right)=\phi(q \otimes p) q^{\prime} .
$$

This context is said to be surjective if $\theta$ and $\phi$ are surjective, and unitary if the biacts ${ }_{S} P_{T}$ and ${ }_{T} Q_{S}$ are unitary.

Example 1.2. Let $S$ be a semigroup and let $E$ be the set of its idempotents. Then we have a unitary Morita context $\left(S, E S E,{ }_{S} S E_{E S E}, E_{S E} E S_{S}, \theta, \phi\right)$ where

$$
\begin{gathered}
\theta: S E \otimes E S \rightarrow S, \quad s e \otimes e^{\prime} s^{\prime} \mapsto s e e^{\prime} s^{\prime}, \\
\phi: E S \otimes S E \rightarrow E S E, \quad e s \otimes s^{\prime} e^{\prime} \mapsto e s s^{\prime} e^{\prime} .
\end{gathered}
$$

If $S=S E S$ then this context is also surjective.

Acceptable Morita contexts for semigroups were introduced in [1] as non-additive analogues of acceptable Morita contexts of rings (see [8]). The existence of an acceptable Morita context with an extra condition guarantees Morita equivalence of the semigroups in that context (see [1, Theorem 2.4]). While unitary surjective Morita contexts can exist only between factorisable semigroups, acceptable Morita contexts can also connect nonfactorisable semigroups (see Example 1.4 and Example 3.8) giving another possibility (besides the equivalence of categories) for developing Morita theory for arbitrary semigroups.

Definition 1.3. A Morita context $\left(S, T,{ }_{S} P_{T},{ }_{T} Q_{S}, \theta, \phi\right)$ is said to be right acceptable if

(1) for every sequence $\left(s_{m}\right)_{m \in \mathbb{N}} \in S^{\mathbb{N}}$, there exists $m_{0} \in \mathbb{N}$ such that $s_{m_{0}} \ldots s_{2} s_{1} \in \operatorname{im}(\theta)$,

(2) for every sequence $\left(t_{m}\right)_{m \in \mathbb{N}} \in T^{\mathbb{N}}$, there exists $m_{0} \in \mathbb{N}$ such that $t_{m_{0}} \ldots t_{2} t_{1} \in \operatorname{im}(\phi)$.

Left acceptable Morita contexts are defined dually. A Morita context is acceptable if it is both right and left acceptable.

Clearly, every surjective Morita context is acceptable. The converse does not hold. 
Example 1.4. Let $n \geq 2, S=\left\langle s \mid s^{n}=s^{n+1}\right\rangle$ and let $T=\{0\}$ where $0:=s^{n}$. Then $T$ is an ideal of the semigroup $S$ and we have a Morita context with homomorphisms

$$
\begin{gathered}
\theta:{ }_{S} S_{T} \otimes{ }_{T} T_{S} \rightarrow{ }_{S} S_{S}, \quad s \otimes 0 \mapsto 0, \\
\phi:{ }_{T} T_{S} \otimes{ }_{S} S_{T} \rightarrow{ }_{T} T_{T}, \quad 0 \otimes s \mapsto 0,
\end{gathered}
$$

where $\phi$ is surjective, but $\theta$ is not. However, this context is acceptable.

Definition 1.5. Semigroups $S$ and $T$ are said to be

- strongly Morita equivalent [10] if they are contained in a unitary surjective Morita context,

- Morita equivalent if the category of firm right $S$-acts is equivalent to the category of firm right $T$-acts.

Strongly Morita equivalent semigroups are necessarily factorisable. In fact, on the class of factorisable semigroups Morita equivalence coincides with strong Morita equivalence [5, Theorem 4.11].

\section{The quantale of unitary ideals}

In this section we study the lattice of unitary ideals of a semigroup. Some results will be formulated in the more general situation of unitary biacts.

We denote the set of all unitary sub-biacts of a biact ${ }_{S} X_{T}$ by $\operatorname{USub}\left({ }_{S} X_{T}\right)$. This is a poset with respect to inclusion where the empty sub-biact is the bottom element. Every biact ${ }_{S} X_{T}$ has also a largest unitary sub-biact: the union of all its unitary sub-biacts, which will be denoted by $\mathrm{U}\left({ }_{S} X_{T}\right)$ or just $\mathrm{U}(X)$. Similar considerations hold for right (or left) acts.

Lemma 2.1. An element $x$ belongs to $\mathrm{U}\left({ }_{S} X_{T}\right)$ if and only if there exist elements $s_{i} \in S, t_{i} \in T$, and $x_{i}, y_{i} \in X$ such that

$$
x=s_{1} x_{1}, x_{1}=y_{1} t_{1}, y_{1}=s_{2} x_{2}, x_{2}=y_{2} t_{2}, \ldots .
$$

Proof. Let $V$ denote the set of all elements $x$ for which the above equalities hold. Then the inclusion $\mathrm{U}\left({ }_{S} X_{T}\right) \subseteq V$ holds because $\mathrm{U}\left({ }_{S} X_{T}\right)$ is unitary.

Conversely, consider an arbitrary element $x \in V$ with the above equalities. For every $s \in S$ and $t \in T$ we have $s x \in V$ because of the equalities

$$
s x=\left(s s_{1}\right) x_{1}, x_{1}=y_{1} t_{1}, y_{1}=s_{2} x_{2}, x_{2}=y_{2} t_{2}, \ldots
$$

and $x t \in X$ because of the equalities

$$
x t=s_{1}\left(x_{1} t\right), x_{1} t=y_{1}\left(t_{1} t\right), y_{1}=s_{2} x_{2}, x_{2}=y_{2} t_{2}, \ldots .
$$

Hence $V$ is a sub-biact of ${ }_{S} X_{T}$.

The equality $V=V T$ follows from the fact that $x=\left(s_{1} y_{1}\right) t_{1}$ and $s_{1} y_{1} \in$ $V$ because of the equalities

$$
s_{1} y_{1}=\left(s_{1} s_{2}\right) x_{2}, x_{2}=y_{2} t_{2}, y_{2}=s_{3} x_{3}, \ldots .
$$

Similarly, we have $V=S V$ because $x=s_{1} x_{1}$ and $x_{1} \in V$ due to

$$
x_{1}=y_{1} t_{1}=s_{2}\left(x_{2} t_{1}\right), x_{2} t_{1}=y_{2}\left(t_{2} t_{1}\right), y_{2}=s_{3} x_{3}, \ldots .
$$


Thus $V$ is a unitary sub-biact, hence it must be contained in $\mathrm{U}\left({ }_{S} X_{T}\right)$.

Corollary 2.2. If $S$ and $T$ are factorisable semigroups then $\mathrm{U}\left({ }_{S} X_{T}\right)=S X T$.

Proof. Clearly, $S X T$ is a unitary sub-biact of ${ }_{S} X_{T}$, so $S X T \subseteq \mathrm{U}\left({ }_{S} X_{T}\right)$.

Conversely, for an element $x \in \mathrm{U}\left({ }_{S} X_{T}\right)$ consider the equalities given by Lemma 2.1. Then $x=s_{1} x_{1}=s_{1} y_{1} t_{1} \in S X T$.

Remark 2.3. The equalities in Lemma 2.1 can be described by an infinite tree as in Figure 1 where each parent is the product of its descendants.

Since the union of unitary sub-biacts is also a unitary sub-biact, the poset $\operatorname{USub}\left({ }_{S} X_{T}\right)$ is a complete lattice where joins are unions. If $A_{i}, i \in I$, are unitary subacts of ${ }_{S} X_{T}$ then it can be checked that

$$
\bigwedge_{i \in I} A_{i}=\mathrm{U}\left(\cap_{i \in I} A_{i}\right)
$$

in the lattice $\operatorname{USub}\left({ }_{S} X_{T}\right)$.

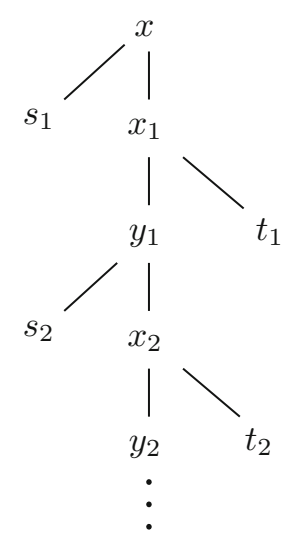

Figure 1. A tree for $x \in \mathrm{U}\left({ }_{S} X_{T}\right)$

Proposition 2.4. For any semigroups $S, T$ and any biact ${ }_{S} X_{T}$, the lattice $\operatorname{USub}\left({ }_{S} X_{T}\right)$ is distributive.

Proof. For any unitary sub-biacts $A, B, C \in \operatorname{USub}\left({ }_{S} X_{T}\right)$ we have to prove the inequality $A \wedge(B \vee C) \leq(A \wedge B) \vee(A \wedge C)$, that is,

$$
\mathrm{U}(A \cap(B \cup C)) \subseteq \mathrm{U}(A \cap B) \cup \mathrm{U}(A \cap C) .
$$

If $x \in \mathrm{U}(A \cap(B \cup C))$ then by Lemma 2.1 there exist $s_{i} \in S, t_{i} \in T$ and $x_{i}, y_{i} \in A \cap(B \cup C)$ such that

$$
x=s_{1} x_{1}, x_{1}=y_{1} t_{1}, y_{1}=s_{2} x_{2}, x_{2}=y_{2} t_{2}, \ldots .
$$

In particular, every $y_{i}$ belongs to $B$ or $C$. Note also that, for every $n \in \mathbb{N}$, we have

$$
y_{n}=s_{n+1} x_{n+1}=s_{n+1} y_{n+1} t_{n+1} .
$$

There are two possibilities. 
(1) The set $\left\{i \in \mathbb{N} \mid y_{i} \in B\right\}$ is infinite. Take an arbitrary $k \in \mathbb{N}$. Then there exists $l \in \mathbb{N}$ such that $y_{k+l} \in B$. Now

$$
y_{k}=s_{k+1} y_{k+1} t_{k+1}=\cdots=s_{k+1} \ldots s_{k+l} y_{k+l} t_{k+l} \ldots t_{k+1}
$$

implies that $y_{k} \in B$, because $B$ is a sub-biact of ${ }_{S} X_{T}$. Also $x_{k} \in B$ because $x_{k}=y_{k} t_{k}$, and thus $x_{k}, y_{k} \in A \cap B$. By Lemma 2.1 this means that $x \in \mathrm{U}(A \cap B)$.

(2) The set $\left\{i \in \mathbb{N} \mid y_{i} \in C\right\}$ is infinite. Then a similar argument shows that $x \in \mathrm{U}(A \cap C)$.

A semigroup $S$ is said to have weak local units if, for every $s \in S$, there exist $u, v \in S$ such that $s=u s=s v$.

Proposition 2.5. If $S$ and $T$ are semigroups with weak local units and ${ }_{S} X_{T}$ is a biact then the lattice $\operatorname{USub}\left({ }_{S} X_{T}\right)$ is algebraic.

Proof. As mentioned before, this lattice is complete. It is straightforward to check that the compact elements of the lattice $\operatorname{USub}\left({ }_{S} X_{T}\right)$ are the finitely generated unitary sub-biacts of ${ }_{S} X_{T}$. If $A \in \operatorname{USub}\left({ }_{S} X_{T}\right)$ then each element $a$ of $A$ can be written as $a=s a=a t$ for some $s \in S, t \in T$, hence the 1-generated sub-biact $S^{1} a T^{1}$ is unitary. Now $A$ is the join of the compact elements $S^{1} a T^{1}$, $a \in A$.

Now we turn our attention to unitary ideals.

Definition 2.6. We say that an ideal $I$ of a semigroup $S$ is right (resp. left) unitary if the $S$-act $I_{S}$ (resp. $\left.{ }_{S} I\right)$ is unitary, that is, $I S=I(\operatorname{resp}$. $S I=I)$. An ideal is called unitary if it is both right and left unitary.

We denote the set of all unitary ideals of a semigroup $S$ by $\operatorname{UId}(S)$. Every semigroup has the unitary ideal $\emptyset$. If $S$ is factorisable then $S$ is also a unitary ideal of itself. If a semigroup has a zero element 0 then $\{0\}$ is a unitary ideal.

Example 2.7. There exist ideals which are not unitary, so for a semigroup $S$ the inclusion $\operatorname{UId}(S) \subseteq \operatorname{Id}(S)$ may be proper. For example, the ideals of the 4-element firm semigroup $S=\{0,1,2,3\}$ with the Cayley table

$\begin{array}{llll}0 & 0 & 0 & 0 \\ 0 & 0 & 0 & 0 \\ 0 & 0 & 0 & 2 \\ 0 & 1 & 2 & 3\end{array}$

are

$$
\emptyset,\{0\},\{0,2\}, S,\{0,1\},\{0,1,2\},
$$

of which the last two are not unitary.

From the two-sided analogue of Proposition 2.4 of [9] we have the following.

Corollary 2.8. If $S$ is a semigroup with weak local units then all ideals of $S$ are unitary. 
Actually, even more is true.

Proposition 2.9. A semigroup has weak right local units if and only if all its right ideals are unitary.

Proof. Straightforward.

Since $\operatorname{UId}(S)=\operatorname{USub}\left({ }_{S} S_{S}\right)$, the above results about biacts apply. So we know that $\operatorname{UId}(S)$ is a complete distributive lattice.

Recall that a quantale is a complete lattice $L$ with an associative binary operation $*$ (called multiplication) such that

$$
x *\left(\bigvee_{k \in K} y_{k}\right)=\bigvee_{k \in K}\left(x * y_{k}\right) \text { and }\left(\bigvee_{k \in K} y_{k}\right) * x=\bigvee_{k \in K}\left(y_{k} * x\right)
$$

for all $x, y_{k} \in L, k \in K$ (where $K$ is any index set). A quantale $L$ is said to be unital if the semigroup $(L, *)$ is a monoid. Quantale homomorphisms are mappings between quantales that preserve multiplication and joins. A homomorphism of unital quantales has to preserve also the identity element.

The poset of unitary ideals of any semigroup has the structure of a quantale.

Proposition 2.10. If $S$ is a semigroup then $\operatorname{UId}(S)$ is a quantale.

Proof. As mentioned earlier, the poset $(\operatorname{UId}(S), \subseteq)$ is a complete lattice where joins are unions. If $I$ and $J$ are unitary ideals of $S$ then also the set

$$
I J=\{i j \mid i \in I, j \in J\}
$$

is a unitary ideal and the set $\operatorname{UId}(S)$ is a semigroup with respect to the multiplication $(I, J) \mapsto I J$. It is also clear that

$$
I J=\bigcup_{k \in K} I_{k} J \text { and } J I=\bigcup_{k \in K} J I_{k}
$$

where $I=\cup_{k \in K} I_{k}$ and $J, I_{k}, k \in K$, are unitary ideals of $S$. Hence $\operatorname{UId}(S)$ is a quantale.

Remark 2.11. A similar proof shows that also $\operatorname{Id}(S)$ is a quantale, where joins and product are defined in the same way as in $\operatorname{UId}(S)$, but meet is intersection.

Although $\operatorname{UId}(S)$ is a subset of $\operatorname{Id}(S)$, it is not a sublattice in general, because meet is computed in different ways in these lattices. However, $\operatorname{UId}(S)$ is a subquantale of $\operatorname{Id}(S)$.

Before formulating the next result note that every semigroup $S$ has a largest factorisable subsemigroup, which we denote by $\mathcal{U}(S)$. It is possible to prove that $\mathcal{U}(S)$ is actually an ideal of $S[6]$.

Proposition 2.12. Let $S$ be a semigroup. The quantale $\operatorname{UId}(S)$ is unital if and only if $\mathcal{U}(S)$ is its identity element. 
Proof. Necessity. Suppose that $\operatorname{UId}(S)$ has an identity element $I$ with respect to multiplication. Then $I I=I$, meaning that $I$ is a factorisable subsemigroup of $S$. Therefore $I$ lies in the largest factorisable subsemigroup $\mathcal{U}(S)$ of $S$. Since $\mathcal{U}(S)$ is factorisable and an ideal, we have $\mathcal{U}(S) \in \operatorname{UId}(S)$. Additionally, for all $J \in \operatorname{UId}(S)$ we have

$$
J=I J \subseteq \mathcal{U}(S) J \subseteq J,
$$

whence $J=\mathcal{U}(S) J$, and similarly $J=J \mathcal{U}(S)$. Thus $\mathcal{U}(S)$ is the identity element of the semigroup $\operatorname{UId}(S)$ and we conclude that $I=\mathcal{U}(S)$.

Sufficiency is clear.

A unital quantale is said to be integral if the multiplicative identity is the top element of the underlying lattice.

Corollary 2.13. If $S$ is a factorisable semigroup then $\operatorname{UId}(S)$ is an integral quantale.

Proof. If $S$ is factorisable then $\mathcal{U}(S)=S$ and $J S=J=S J$ for every unitary ideal $J$.

\section{Unitary ideals and Morita contexts}

Recall the following result about ideal lattices.

Proposition 3.1 [4]. If $S$ and $T$ are Morita equivalent semigroups with weak local units then their ideal lattices are isomorphic.

Combining this result with Proposition 2.2 from [9] one can even say that the quantales of the ideals of such $S$ and $T$ are isomorphic.

The following example shows that Proposition 3.1 does not generalise to the class of firm semigroups.

Example 3.2 (Ideal lattices of Morita equivalent firm semigroups need not be isomorphic). Let $T=\{0,1,2,3\}$ be the semigroup with the Cayley table

$\begin{array}{llll}0 & 0 & 0 & 3 \\ 0 & 0 & 1 & 3 \\ 0 & 0 & 2 & 3 \\ 3 & 3 & 3 & 0\end{array}$.

This is a factorisable semigroup with a commutative factorisable subsemigroup $S=\{0,2,3\}$. Since 2 is a right identity element of $T$ and $S$, it follows immediately that these two semigroups are firm. We will show that $T$ is an enlargement of $S$. This implies that they are strongly Morita equivalent as shown in the beginning of Section 2 in [7].

Since

$$
\begin{aligned}
& 0=0 \cdot 0 \cdot 0, \\
& 1=1 \cdot 2 \cdot 2, \\
& 2=2 \cdot 2 \cdot 2, \\
& 3=0 \cdot 0 \cdot 3,
\end{aligned}
$$


we have the inclusion $T \subseteq T S T$. The opposite inclusion being obvious, we conclude that $T=T S T$.

Factorisability of $S$ implies $S=S S S \subseteq S T S$. Since $T S=T$, we see that $S T S=S T \subseteq\{0,2,3\}=S$. Thus also $S=S T S$ and we have proved that $T$ is an enlargement of $S$.

The ideal lattices

$$
\begin{aligned}
& \operatorname{Id}(S)=\{\emptyset,\{0,3\}, S\}, \\
& \operatorname{Id}(T)=\{\emptyset,\{0,3\},\{0,1,3\}, T\}
\end{aligned}
$$

of the (strongly) Morita equivalent semigroups $S$ and $T$ are not isomorphic.

Our next aim is to show that under relatively weak assumptions two semigroups will have isomorphic quantales of unitary ideals. For this we will need the following lemma.

Lemma 3.3. If $\Gamma=\left(S, T,{ }_{S} P_{T},{ }_{T} Q_{S}, \theta, \phi\right)$ is an acceptable Morita context and $B_{T}$ is a unitary $T$-act then $B=B \operatorname{im}(\phi)$.

Proof. Take $b_{0} \in B$. Since $B_{T}$ is unitary, we can find sequences $\left(b_{k}\right)_{k \in \mathbb{N}} \in B^{\mathbb{N}}$ and $\left(t_{k}\right)_{k \in \mathbb{N}} \in T^{\mathbb{N}}$ such that $b_{k}=b_{k+1} t_{k+1}$ for every $k \geq 0$. Acceptability of $\Gamma$ implies that there exists $n \in \mathbb{N}$ such that $t_{n} \ldots t_{2} t_{1}=\phi(q \otimes p)$ for some $p \in P$ and $q \in Q$, hence

$$
b_{0}=b_{1} t_{1}=b_{2} t_{2} t_{1}=\cdots=b_{n} t_{n} \ldots t_{2} t_{1}=b_{n} \phi(q \otimes p) \in B \operatorname{im}(\phi) .
$$

Thus $B \subseteq B \operatorname{im}(\phi)$. The opposite inclusion is obvious.

Now we can prove our main result.

Theorem 3.4. If two semigroups are connected by an acceptable Morita context then there exists an isomorphism between the quantales of their unitary ideals. This isomorphism takes finitely generated ideals to finitely generated ideals and principal ideals to principal ideals. If the quantales are unital then this isomorphism is an isomorphism of unital quantales.

Proof. 1. Let $\Gamma=\left(S, T,{ }_{S} P_{T},{ }_{T} Q_{S}, \theta, \phi\right)$ be an acceptable Morita context connecting semigroups $S$ and $T$. For any unitary ideal $J$ of $T$ we see that the set $\theta(P J \otimes Q)=\{\theta(p j \otimes q) \mid p \in P, j \in J, q \in Q\}$ is an ideal of $S$ because $\theta$ is a homomorphism of $(S, S)$-biacts. We will prove that it is a unitary ideal. Using Lemma 3.3 we can calculate

$$
\begin{aligned}
\theta(P J \otimes Q) & =\theta(P J \operatorname{im}(\phi) \otimes Q)=\theta(P J \otimes \operatorname{im}(\phi) Q)=\theta(P J \otimes Q \operatorname{im}(\theta)) \\
& =\theta(P J \otimes Q) \operatorname{im}(\theta) \subseteq \theta(P J \otimes Q) S=\theta(P J \otimes Q S) \subseteq \theta(P J \otimes Q) .
\end{aligned}
$$

Thus $\theta(P J \otimes Q)=\theta(P J \otimes Q) S$ and it is a unitary right ideal of $S$. Similar calculations show that it is left unitary.

Analogously $\phi(Q I \otimes P)$ is a unitary ideal of $T$ for every unitary ideal $I$ of $S$.

This allows to consider the mappings

$$
\begin{aligned}
& \Phi: \operatorname{UId}(S) \rightarrow \operatorname{UId}(T), \Phi(I):=\phi(Q I \otimes P), \\
& \Theta: \operatorname{UId}(T) \rightarrow \operatorname{UId}(S), \Theta(J):=\theta(P J \otimes Q) .
\end{aligned}
$$


Then, precisely as in the proof of Theorem 3 in [4], one can show that $\Theta$ and $\Phi$ are inverses of each other. They also preserve order and hence they are isomorphisms of complete lattices. Moreover, for arbitrary $I_{1}, I_{2} \in \operatorname{UId}(S)$ we have

$$
\begin{aligned}
\Phi\left(I_{1}\right) \Phi\left(I_{2}\right) & =\phi\left(Q I_{1} \otimes P\right) \phi\left(Q I_{2} \otimes P\right)=\phi\left(Q I_{1} \otimes P \phi\left(Q I_{2} \otimes P\right)\right) \\
& =\phi\left(Q I_{1} \otimes \theta\left(P \otimes Q I_{2}\right) P\right)=\phi\left(Q I_{1} \otimes \theta(P \otimes Q) I_{2} P\right) \\
& =\phi\left(Q I_{1} \otimes i m(\theta) I_{2} P\right)=\phi\left(Q I_{1} \otimes I_{2} P\right)=\phi\left(Q I_{1} I_{2} \otimes P\right)=\Phi\left(I_{1} I_{2}\right)
\end{aligned}
$$

where $\operatorname{im}(\theta) I_{2}=I_{2}$ by the dual of Lemma 3.3. Similarly, $\Theta\left(J_{1}\right) \Theta\left(J_{2}\right)=$ $\Theta\left(J_{1} J_{2}\right)$ for arbitrary $J_{1}, J_{2} \in \operatorname{UId}(T)$. Thus $\Phi$ and $\Theta$ are isomorphisms of quantales.

2. We prove the claim about finitely generated ideals. Obviously, $\Phi(\emptyset)=\emptyset$ and $\Theta(\emptyset)=\emptyset$.

Take a unitary finitely generated ideal $I=\bigcup_{j=1}^{n} S^{1} a_{j} S^{1}$ of $S$ and $i \in$ $\{1, \ldots, n\}$. AS $a_{i} \in I$ and $I=I \mathrm{im}(\theta)$, we can write $a_{i}=s_{k_{i}} a_{k_{i}} \theta\left(p_{k_{i}} \otimes q_{k_{i}}\right)$ for some $k_{i} \in\{1, \ldots, n\}, s_{k_{i}} \in S^{1}, p_{k_{i}} \in P$ and $q_{k_{i}} \in Q$. From $I=\operatorname{im}(\theta) I$ it follows that $a_{k_{i}}=\theta\left(p_{l_{i}} \otimes q_{l_{i}}\right) a_{l_{i}} z_{l_{i}}$ for some $l_{i} \in\{1, \ldots, n\}, z_{l_{i}} \in S^{1}, p_{l_{i}} \in P$ and $q_{l_{i}} \in Q$. Hence

$$
a_{i}=s_{k_{i}} \theta\left(p_{l_{i}} \otimes q_{l_{i}}\right) a_{l_{i}} z_{l_{i}} \theta\left(p_{k_{i}} \otimes q_{k_{i}}\right) .
$$

Now, for any $p \in P$ and $q \in Q$ we have

$$
\begin{aligned}
\phi\left(q a_{i} \otimes p\right) & =\phi\left(q \theta\left(s_{k_{i}} p_{l_{i}} \otimes q_{l_{i}}\right) a_{l_{i}} \theta\left(z_{l_{i}} p_{k_{i}} \otimes q_{k_{i}}\right) \otimes p\right) \\
& =\phi\left(\phi\left(q \otimes s_{k_{i}} p_{l_{i}}\right) \phi\left(q_{l_{i}} a_{l_{i}} \otimes z_{l_{i}} p_{k_{i}}\right) q_{k_{i}} \otimes p\right) \\
& =\phi\left(q \otimes s_{k_{i}} p_{l_{i}}\right) \phi\left(q_{l_{i}} a_{l_{i}} \otimes z_{l_{i}} p_{k_{i}}\right) \phi\left(q_{k_{i}} \otimes p\right) \\
& \subseteq T^{1} \phi\left(q_{l_{i}} a_{l_{i}} \otimes z_{l_{i}} p_{k_{i}}\right) T^{1} .
\end{aligned}
$$

Therefore

$$
\begin{aligned}
\Phi(I) & =\left\{\phi\left(q a_{i} \otimes p\right) \mid p \in P, q \in Q, i \in\{1, \ldots, n\}\right\} \\
& \subseteq \bigcup_{j=1}^{n} T^{1} \phi\left(q_{l_{i}} a_{l_{i}} \otimes p_{k_{i}}\right) T^{1} .
\end{aligned}
$$

The opposite inclusion being clear, we conclude that

$$
\Phi(I)=\bigcup_{j=1}^{n} T^{1} \phi\left(q_{l_{i}} a_{l_{i}} \otimes p_{k_{i}}\right) T^{1} .
$$

3. The relation above for $n=1$ tells us that $\Phi$ maps unitary principal ideals to unitary principal ideals.

4. If $\operatorname{UId}(S)$ and $\operatorname{UId}(T)$ are unital quantales then by Proposition 2.12 their identity elements are $\mathcal{U}(S)$ and $\mathcal{U}(T)$. Note that $\mathcal{U}(S)$ is an idempotent in the quantale $\operatorname{UId}(S)$ such that $I \subseteq \mathcal{U}(S)$ for every other idempotent $I$. A similar statement is true for $\mathcal{U}(T)$. Since the quantale isomorphisms preserve idempotents and order, it follows that $\Phi(\mathcal{U}(S))=\mathcal{U}(T)$ and $\Theta(\mathcal{U}(T))=\mathcal{U}(S)$. So $\Phi$ and $\Theta$ are isomorphisms of unital quantales. 
Corollary 3.5. The quantales of unitary ideals of strongly Morita equivalent semigroups are isomorphic.

Proof. Two strongly Morita equivalent semigroups are contained in a unitary surjective Morita context which, of course, is acceptable.

Corollary 3.6. If two strongly Morita equivalent semigroups have weak local units then their quantales of ideals are isomorphic.

Proof. This follows from Theorem 3.4 and Proposition 2.9.

As an application of Theorem 3.4 we point out the following.

Example 3.7. Let $\mathcal{M}$ be a factorisable Rees matrix semigroup over a factorisable semigroup $S$. By Proposition 2 in [3] the semigroups $S$ and $\mathcal{M}$ are strongly Morita equivalent. Hence they have isomorphic quantales of unitary ideals.

Example 3.8. Let $S$ be an arbitrary semigroup, $n$ a natural number, and let us denote $S^{n}=\left\{s_{1} s_{2} \ldots s_{n} \mid s_{1}, s_{2}, \ldots, s_{n} \in S\right\}$. Then we have an acceptable Morita context $\left(S, S^{n}, S_{S} S_{S^{n}}, S^{n} S_{S}, \theta, \phi\right)$ where

$$
\begin{gathered}
\theta: S \otimes_{S^{n}} S \rightarrow S, \quad s \otimes s^{\prime} \mapsto s s^{\prime}, \\
\phi: S \otimes_{S} S \rightarrow S^{n}, \quad s \otimes s^{\prime} \mapsto s s^{\prime} .
\end{gathered}
$$

Hence $\operatorname{UId}(S)$ and $\operatorname{UId}\left(S^{n}\right)$ are isomorphic quantales. It follows that if $S$ is an inflation of its subsemigroup $T$ then $\operatorname{UId}(S)$ is isomorphic to $\operatorname{UId}(T)$ because $S^{2}=T^{2}$.

We also have the following analogue of Theorem 2.5 in [9].

Theorem 3.9. If two semigroups $S$ and $T$ are connected by an acceptable Morita context $\left(S, T,{ }_{S} P_{T},{ }_{T} Q_{S}, \theta, \phi\right)$ then the following lattices are isomorphic:

(1) $\operatorname{UId}(S)$

(2) $\operatorname{UId}(T)$,

(3) $\operatorname{USub}\left({ }_{S} P_{T}\right)$,

(4) $\operatorname{USub}\left({ }_{T} Q_{S}\right)$.

Proof. We only prove the isomorphism of (1) and (3) (the isomorphism of (2) and (4) is similar and the isomorphism of (1) and (2) is proved in Theorem 3.4).

Observe that if $I \in \operatorname{UId}(S)$ then $I P$ is a sub-biact of ${ }_{S} P_{T}$. We need to show that it is unitary. Since $I=S I$, we have $I P=S(I P)$. The inclusion $I P T \subseteq I P$ is clear. We have to prove the inclusion $I P \subseteq I P T$. Take an element $i_{0} p_{0} \in I P$. Using the equality $I=I S$ we can find sequences $\left(i_{k}\right)_{k \in \mathbb{N}} \in I^{\mathbb{N}}$ and $\left(s_{k}\right)_{k \in \mathbb{N}} \in S^{\mathbb{N}}$ such that $i_{k}=i_{k+1} s_{k+1}$ for every $k \geq 0$. Since the context is acceptable, there exist $n \in \mathbb{N}, p \in P, q \in Q$ such that $s_{n} \ldots s_{2} s_{1}=\theta(p \otimes q)$. Now

$$
i_{0} p_{0}=i_{n} s_{n} \ldots s_{2} s_{1} p_{0}=i_{n} \theta(p \otimes q) p_{0}=i_{n} p \phi\left(q \otimes p_{0}\right) \in I P T .
$$

Thus the equality $I P=(I P) T$ also holds and $I P \in \operatorname{USub}\left({ }_{S} P_{T}\right)$. 
If $A \in \operatorname{USub}\left({ }_{S} P_{T}\right)$ then using Lemma 3.3 we obtain

$$
\begin{aligned}
\theta(A \otimes Q) & =\theta(A \operatorname{im}(\phi) \otimes Q)=\theta(A \phi(Q \otimes P) \otimes Q) \\
& =\theta(A \otimes \phi(Q \otimes P) Q)=\theta(A \otimes Q \theta(P \otimes Q))=\theta(A \otimes Q) \operatorname{im}(\theta) \\
& \subseteq \theta(A \otimes Q) S=\theta(A \otimes Q S) \subseteq \theta(A \otimes Q),
\end{aligned}
$$

whence $\theta(A \otimes Q)=\theta(A \otimes Q) S$. Also, $\theta(A \otimes Q)=\theta(S A \otimes Q)=S \theta(A \otimes Q)$, and so $\theta(A \otimes Q) \in \operatorname{UId}(S)$.

This allows to consider the mappings

$$
\begin{aligned}
& \Psi: \operatorname{UId}(S) \rightarrow \operatorname{USub}\left({ }_{S} P_{T}\right), \Psi(I):=I P, \\
& \Omega: \operatorname{USub}\left({ }_{S} P_{T}\right) \rightarrow \operatorname{UId}(S), \Omega(A):=\theta(A \otimes Q) .
\end{aligned}
$$

These mappings preserve order, and they are inverses of each other because, by Lemma 3.3,

$$
\begin{aligned}
& (\Omega \Psi)(I)=\theta(I P \otimes Q)=I \theta(P \otimes Q)=I \operatorname{im}(\theta)=I, \\
& (\Psi \Omega)(A)=\theta(A \otimes Q) P=A \phi(Q \otimes P)=A \operatorname{im}(\phi)=A .
\end{aligned}
$$

An ideal $I$ of a semigroup $S$ is said to be factorisable if it is factorisable as a semigroup (that is, the equality $I^{2}=I$ holds).

Every factorisable ideal is unitary. The converse does not hold.

Example 3.10 (A unitary ideal need not be factorisable). Let $X^{+}$and $X^{*}$ be the free semigroup and the free monoid on an alphabet $X$, respectively. Then $X^{+}$is a unitary non-factorisable ideal of $X^{*}$.

The empty ideal is factorisable and any union of factorisable ideals of a given semigroup is a factorisable ideal. Hence the set of factorisable ideals is a complete lattice.

Proposition 3.11. Let $S$ and $T$ be arbitrary semigroups connected by an acceptable Morita context $\left(S, T,{ }_{S} P_{T},{ }_{T} Q_{S}, \theta, \phi\right)$ and let $I \subseteq S, J \subseteq T$ be unitary ideals that correspond to each other under the isomorphism of Theorem 3.4. Then, if one of $I$ and $J$ is factorisable, so is the other and they are (strongly) Morita equivalent as semigroups. In particular, $\mathcal{U}(S)$ and $\mathcal{U}(T)$ are strongly Morita equivalent.

Proof. By our assumptions $I=\Theta(J)=\theta(P J \otimes Q)$. Since, in particular, $\Theta$ is a semigroup homomorphism, one of $I$ and $J$ is factorisable precisely when the other is.

Let now $I$ and $J$ be factorisable ideals. It is easy to see that $\Gamma^{\prime}=$ $\left(I, J, I P J, J Q I, \theta^{\prime}, \phi^{\prime}\right)$, where the biact homomorphisms $\theta^{\prime}$ and $\phi^{\prime}$ are defined by

$$
\begin{aligned}
\theta^{\prime}\left(i p j \otimes j^{\prime} q i^{\prime}\right) & :=\theta\left(i p j \otimes j^{\prime} q i^{\prime}\right), \\
\phi^{\prime}\left(j^{\prime} q i^{\prime} \otimes i p j\right) & :=\phi\left(j^{\prime} q i^{\prime} \otimes i p j\right),
\end{aligned}
$$

is a unitary Morita context. Note that $\theta^{\prime}$ and $\phi^{\prime}$ are well defined because the mappings

$$
\begin{aligned}
& I P J \times J Q I \rightarrow I, \quad\left(i p j, j^{\prime} q i^{\prime}\right) \mapsto \theta\left(i p j \otimes j^{\prime} q i^{\prime}\right), \\
& J Q I \times I P J \rightarrow J, \quad\left(j^{\prime} q i^{\prime}, i p j\right) \mapsto \phi\left(j^{\prime} q i^{\prime} \otimes i p j\right)
\end{aligned}
$$


are balanced.

The map $\theta^{\prime}$ is surjective, since

$$
\begin{aligned}
\theta^{\prime}(I P J \otimes J Q I) & =\theta(I P J \otimes J Q I)=\theta(I P J J \otimes Q I) \\
& =\theta(I P J \otimes Q I)=I \theta(P J \otimes Q) I=I I I=I,
\end{aligned}
$$

and $\phi^{\prime}$ can be shown to be surjective in an analogous way. Hence $I$ and $J$ are (strongly) Morita equivalent semigroups.

As explained in the last part of the proof of Theorem 3.4, the factorisable ideals $\mathcal{U}(S)$ and $\mathcal{U}(T)$ correspond to each other under the isomorphisms $\Phi$ and $\Theta$.

Corollary 3.12. If two semigroups are connected by an acceptable Morita context then their lattices of factorisable ideals are isomorphic.

Proof. By Proposition 3.11 the mappings $\Theta$ and $\Phi$ restrict to mutually inverse isomorphisms between the lattices of factorisable ideals.

Theorem 3.4 does not hold for one-sided ideals.

Example 3.13 (The lattices of (unitary) right ideals of Morita equivalent semigroups with local units need not be isomorphic). If $S=\{1\}$ and $T$ is a nonsingleton rectangular band then they have non-isomorphic lattices of (unitary) right ideals, although $S$ is a monoid and $T$ has local units. These semigroups are strongly Morita equivalent by Theorem 16 in [2].

One may also consider the posets of firm ideals. An ideal $I$ of a semigroup $S$ is said to be firm if it is firm as a right and left $S$-act. The following example shows that, contrary to Corollary 3.5, the posets of firm ideals of strongly Morita equivalent semigroups need not be isomorphic.

Example 3.14. Consider the factorisable semigroup $S=\{0,1,2,3\}$ from Example 2.7 and its commutative subsemigroup $T=\{0,2,3\}$. This semigroup has a left identity element 3 which also belongs to $T$. This easily implies that $S$ is an enlargement of $T$, and therefore $S$ and $T$ are (strongly) Morita equivalent.

The semigroup $T$ has firm ideals $\emptyset,\{0\},\{0,2\}, T$ and the semigroup $S$ has firm ideals $\emptyset,\{0\}, S$. Thus the posets of firm ideals of $S$ and $T$ are not isomorphic.

\section{Acknowledgements}

Thanks are due to Kalle Kaarli for useful comments. Open access funding provided by Alfréd Rényi Institute of Mathematics. 
Open Access. This article is licensed under a Creative Commons Attribution 4.0 International License, which permits use, sharing, adaptation, distribution and reproduction in any medium or format, as long as you give appropriate credit to the original author(s) and the source, provide a link to the Creative Commons licence, and indicate if changes were made. The images or other third party material in this article are included in the article's Creative Commons licence, unless indicated otherwise in a credit line to the material. If material is not included in the article's Creative Commons licence and your intended use is not permitted by statutory regulation or exceeds the permitted use, you will need to obtain permission directly from the copyright holder. To view a copy of this licence, visit http://creativecommons.org/ licenses/by/4.0/.

Publisher's Note Springer Nature remains neutral with regard to jurisdictional claims in published maps and institutional affiliations.

\section{References}

[1] Laan, V.: Acceptable Morita contexts for semigroups. ISRN Algebra vol. 2012, article ID 725627 (2012)

[2] Laan, V.: Context equivalence of semigroups. Period. Math. Hungar. 60, 81-94 (2010)

[3] Laan, V., Márki, L.: Strong Morita equivalence of semigroups with local units. J. Pure Appl. Algebra 215, 2538-2546 (2011)

[4] Laan, V., Márki, L.: Morita invariants for semigroups with local units. Monatsh. Math. 166, 441-451 (2012)

[5] Laan, V., Reimaa, Ü.: Morita equivalence of factorisable semigroups. Int. J. Algebra Comput. 29, 723-741 (2019)

[6] Laan, V., Reimaa, Ü., Tart, L.: $\mathcal{U}$-fair semigroups (2020, preprint)

[7] Lawson, M.V., Márki, L.: Enlargements and coverings by Rees matrix semigroups. Monatsh. Math. 129, 191-195 (2000)

[8] Marín, L.: Morita equivalence based on contexts for various categories of modules over associative rings. J. Pure Appl. Algebra 133, 219-232 (1998)

[9] Sardar, S.K., Gupta, S.: A note on Morita invariants of semigroups. Semigroup Forum 92, 71-76 (2016)

[10] Talwar, S.: Strong Morita equivalence and a generalisation of the Rees theorem. J. Algebra 181, 371-394 (1996) 
Valdis Laan

Institute of Mathematics and Statistics

University of Tartu

51009 Tartu

Estonia

e-mail: valdis.laan@ut.ee

László Márki

Alfréd Rényi Institute of Mathematics

Budapest 1364

Hungary

e-mail: marki.laszlo@renyi.hu

Ülo Reimaa

Institute of Computer Science

University of Tartu

51009 Tartu

Estonia

e-mail: ulo.reimaa@gmail.com

Received: 16 October 2019.

Accepted: 29 February 2020. 